\title{
Spectrum of Permanent's Values and Its Extremal Magnitudes in $\Lambda_{n}^{3}$ and $\Lambda_{n}(\alpha, \beta, \gamma)$
}

\author{
Vladimir Shevelev \\ Department of Mathematics, Ben-Gurion University of the Negev, 84105 Beersheba, Israel \\ Correspondence should be addressed to Vladimir Shevelev; shevelev@bgu.ac.il
}

Received 10 June 2013; Accepted 25 September 2013

Academic Editor: Manlio Gaudioso

Copyright (C) 2013 Vladimir Shevelev. This is an open access article distributed under the Creative Commons Attribution License, which permits unrestricted use, distribution, and reproduction in any medium, provided the original work is properly cited.

Let $\Lambda_{n}^{k}$ denote the class of $(0,1)$ square matrices containing in each row and in each column exactly $k$ l's. The minimal value of $k$, for which the behavior of the permanent in $\Lambda_{n}^{k}$ is not quite studied, is $k=3$. We give a simple algorithm for calculation of upper magnitudes of permanent in $\Lambda_{n}^{3}$ and consider some extremal problems in a generalized class $\Lambda_{n}(\alpha, \beta, \gamma)$, the matrices of which contain in each row and in each column nonzero elements $\alpha, \beta$, and $\gamma$ and $n-3$ zeros.

\section{Introduction}

The definition of permanent of a square matrix $A=\left(a_{i, j}\right)$ of order $n$ is per $A=\sum \prod_{i=1}^{n} a_{i, \sigma(i)}$, where sum is over all permutations $\sigma$ of numbers $1, \ldots, n$. This definition is very combinatorial. For example, if $A$ is $(0,1)$-matrix, then $\operatorname{per} A$ is the number of arrangements of $n$ nonattacking each other rooks on positions of 1's of $A$. Therefore, the most natural applications of the permanent are in combinatorics: enumerating the Latin rectangles and squares, permutations with restricted positions, and different problems in the theory of graphs. Many interesting examples of applications of the permanent one can find in [1], chapter 8 (and not only in Mathematics!). To applications of the permanent there promoted celebrate proofs of the best known van der Waerden's and Minc's conjectures by Egorychev [2], Falikman [3], and Brègman [4], respectively. For example, Alon and Friedland [5] excellently used the Minc-Bregman inequality for permanent of $(0,1)$ matrices for finding the maximum number of perfect matchings in graphs with a given degree sequence.

Let $\Lambda_{n}^{k}$ denote the class of $(0,1)$ square matrices containing in each row and in each column exactly $k$ l's. If $A \in \Lambda_{n}^{k}$, then matrix $k^{-1} A$ is doubly stochastic, since all its row and column sums equal 1. Therefore, $\Lambda_{n}^{k}$-matrices are also called doubly stochastic $(0,1)$-matrices. These matrices have especially simple and attractive structure and have many applications. It is important that especially class $\Lambda_{n}^{3}$ in permanent's history was a nontrivial test area for different researches of the permanent. For example, one can mention a remarkable Merriell research [6] of the maximum of permanent on class $\Lambda_{n}^{3}$. An author's research [7] of the permanent on $\Lambda_{7}^{3}$-matrices (see Section 9) has led to the disproof of an important Balasubramanian conjecture [8] which would yield a full proof of 1967 Ryser hypothesis [9] on transversals of Latin squares.

In the present paper we consider many other problems for $\Lambda_{n}^{3}$. We study also the following natural generalization of $\Lambda_{n}^{3}$. For given real or complex nonzero numbers $\alpha, \beta$, and $\gamma$, denote by $\Lambda_{n}(\alpha, \beta, \gamma)$ the class of square matrices containing every number from $\{\alpha, \beta, \gamma\}$ exactly one time in each row and in each column, such that the other elements are 0's. It is clear that, if $A \in \Lambda_{n}(\alpha, \beta, \gamma)$ and $\alpha+\beta+\gamma \neq 0$, then the matrix $(\alpha+\beta+\gamma)^{-1} A$ is doubly stochastic.

Definition 1. One calls $p$-spectrum in $\Lambda_{n}^{k}$ (denoting it $\left.p s\left[\Lambda_{n}^{k}\right]\right)$ the set of all the values which are taken by the permanent in $\Lambda_{n}^{k}$.

Note that $p$-spectrum in $\Lambda_{n}^{1}$ trivially is $\{1\}$. It is known (cf. Tarakanov [10]) that

$$
\operatorname{ps}\left[\Lambda_{n}^{2}\right]=\left\{2,2^{2}, 2^{3}, \ldots, 2^{\lfloor n / 2\rfloor}\right\} .
$$

But, for $k \geq 3$, p-spectrum of $\Lambda_{n}^{k}$, generally speaking, is unknown. Greenstein (cf. [1], point 8.4, Problem 3) put 
the problem of describing the $p$-spectrum in $\Lambda_{n}^{3}$. In this paper we give an algorithm for calculation of upper values of $p$ spectrum in $\Lambda_{n}^{3}$. We also obtain several results for a generalized class $\Lambda_{n}(\alpha, \beta, \gamma)$ with real nonzero numbers $\alpha, \beta$, and $\gamma$. Some results of the present paper were announced by the author in [11].

\section{What Is Known about $\Lambda_{n}^{3}$ ?}

(1) Explicit formula for $\left|\Lambda_{n}^{3}\right|$ (cf. Stanley [12], chapter 1) is

$$
\left|\Lambda_{n}^{3}\right|=6^{-n} \sum_{k_{1}+k_{2}+k_{3}=n, k_{i} \geq 0} \frac{(-1)^{k_{2}} n !^{2}\left(k_{2}+3 k_{3}\right) ! 2^{k_{1}} 3^{k_{2}}}{k_{1} ! k_{2} ! k_{3} !^{2} 6^{k_{3}}} .
$$

(2) Asymptotic formula for $\left|\Lambda_{n}^{3}\right|$ (cf. O’Neil [13]) is

$$
\left|\Lambda_{n}^{3}\right|=\frac{(3 n) !}{(36)^{n}} e^{-2}\left(1+O\left(n^{-1+\varepsilon}\right)\right),
$$

where $\varepsilon>0$ is arbitrarily small for sufficiently large $n$.

In addition, note that $\left|\Lambda_{n}(\alpha, \beta, \gamma)\right|$ with different $\alpha, \beta$, and $\gamma$ is, evidently, the number of 3-rowed Latin rectangles of length $n$ such that

$$
\left|\Lambda_{n}(\alpha, \beta, \gamma)\right|=n ! K_{n},
$$

where $K_{n}$ is the number of reduced 3-rowed Latin rectangles with the first row $\{1,2, \ldots, n\}$. It is known (Riordan [14], pp. 204-210) that

$$
K_{n}=\sum_{k=0}^{\lfloor n / 2\rfloor}\left(\begin{array}{l}
n \\
k
\end{array}\right) D_{n-k} D_{k} U_{n-2 k}
$$

where $D_{n}$ is subfactorial:

$$
D_{0}=1, \quad D_{n}=n D_{n-1}+(-1)^{n}, \quad n \geq 1,
$$

and $\left\{U_{n}\right\}$ is sequence of Lucas numbers of the Ménage problem which is defined by Cayley recursion (cf. [14], p. 201)

$$
\begin{gathered}
U_{0}=1, \quad U_{1}=-1, \quad U_{2}=0, \\
U_{n}=n U_{n-1}+\frac{n}{n-2} U_{n-2}+4 \frac{(-1)^{n}}{n-2}, \quad n \geq 3
\end{gathered}
$$

(see [15], sequences A102761, A000186).

Denote, furthermore, by $\bar{\Lambda}_{n}^{3}$ the set of matrices in $\Lambda_{n}^{3}$ with 1 's on the main diagonal. Note that

$$
p s\left[\Lambda_{n}^{3}\right]=p s\left[\bar{\Lambda}_{n}^{3}\right] \text {. }
$$

Indeed, it is well known that every $\Lambda_{n}^{3}$-matrix $A$ has a diagonal of ones (i.e., a set of 1's not two in the same row or column). Let $l$ be such a diagonal. There exists a permutation of rows and columns $\pi$ such that $\pi(l)$ will be the main diagonal of $\pi(A)$. Nevertheless, $\operatorname{per}(\pi(A))=\operatorname{per} A$ and $(8)$ follows.

(3) A known explicit formula for $\left|\bar{\Lambda}_{n}^{3}\right|$ (Shevelev [16]) has a close structure to (5):

$$
\left|\bar{\Lambda}_{n}^{3}\right|=\sum_{k=0}^{\lfloor n / 2\rfloor}\left(\begin{array}{l}
n \\
k
\end{array}\right) S_{n-k} S_{k} U_{n-2 k}
$$

where sequence $\left\{S_{n}\right\}$ is defined by recursion

$$
S_{0}=1, \quad S_{1}=0, \quad S_{n}=(n-1)\left(S_{n-1}+\frac{1}{2} S_{n-2}\right), \quad n \geq 2 .
$$

(4) Asymptotic formula for $\left|\bar{\Lambda}_{n}^{3}\right|$ (Shevelev [16]) is

$$
\left|\bar{\Lambda}_{n}^{3}\right|=C \sqrt{n}\left(\frac{n}{e}\right)^{2 n}\left(1+O\left(n^{-1+\varepsilon}\right)\right),
$$

where

$$
C=2 \sqrt{\pi e^{-5}}=0.29098 \ldots
$$

and $\varepsilon>0$ is arbitrarily small for sufficiently large $n$.

(5) Denote by $\widehat{\Lambda}_{n}^{3}$ the set of symmetric matrices in $\bar{\Lambda}_{n}^{3}$. Pspectrum on $\widehat{\Lambda}_{n}^{3}$ is given by the following theorem (Shevelev [11]).

Theorem 2. Let $R(n ; 3)$ denote the set of all partitions of $n$ with parts more than or equal to 3. To every partition $r \in R(n ; 3)$ : $n=n_{1}+n_{2}+\cdots+n_{m}, m=m(r)$, put in a correspondence the number

$$
H(r)=\prod_{i=1}^{m} a\left(n_{i}\right)
$$

where sequence $\{a(n)\}$ is defined by the recursion

$$
\begin{aligned}
& a(3)=6, \quad a(4)=9, \\
& a(n)=a(n-1)+a(n-2)-2, \quad n \geq 5 .
\end{aligned}
$$

Then one has

$$
p s\left[\widehat{\Lambda}_{n}^{3}\right]=\{H(r): r \in R(n ; 3)\} .
$$

(6) The maximal value $M(n)$ of permanent in $\Lambda_{n}^{3}$ was found by Merriell [6].

Theorem 3. If $n \equiv h(\bmod 3), h=0,1,2$, then

$$
M(n)=6^{(n-h) / 3}\left\lfloor\left(\frac{3}{2}\right)^{h}\right\rfloor .
$$

Note that the case $h=0$ of (16) easily follows from the Minc-Bregman inequality for permanent of $(0,1)$-matrices.

(7) Put $M(n)=M^{(1)}(n)$. In case of $n \equiv 0(\bmod 3)$, Bolshakov [17] showed that the second maximal $M^{(2)}<M^{(1)}(n)$ of permanent in $\Lambda_{n}^{3}$ (such that interval $\left(M^{(2)}, M^{(1)}\right.$ ) is free from values of permanent in $\Lambda_{n}^{3}$ ) equals

$$
M^{(2)}(n)= \begin{cases}20, & \text { if } n=6 \\ 120, & \text { if } n=9 \\ \frac{9}{16} 6^{n / 3}, & \text { if } n \geq 12\end{cases}
$$

Note that both $M^{(1)}(n)$ and $M^{(2)}(n)$ are attained in $\widehat{\Lambda}_{n}^{3}$. 
(8) Denote by $m(n)$ the minimal value of permanent in $\Lambda_{n}^{3}$. In 1979, Voorhoeve [18] obtained a beautiful lower estimate for $m(n)$ :

$$
m(n) \geq 6\left(\frac{4}{3}\right)^{n-3} .
$$

This estimate remains the best even after proof by Egorychev [2] and Falikman [3], the famous van der Waerden conjectural lower estimate per $A \geq n ! / n^{n}$ for every $n \times n$ doubly stochastic matrix $A$. Indeed, this estimate yields only $m(n) \geq$ $3^{n}\left(n ! / n^{n}\right)$, such that (18) is stronger for $n \geq 4$.

(9) Bolshakov [19] found $p$-spectrum in $\Lambda_{n}^{3}$ in cases $n \leq 8$. Namely, he added to the evident $p$-spectrums $p s\left[\Lambda_{3}^{3}\right]=\{6\}$ and $p s\left[\Lambda_{4}^{3}\right]=\{9\}$ also the following $p$-spectrums:

$$
\begin{gathered}
\text { ps }\left[\Lambda_{5}^{3}\right]=\{12,13\}, \quad \text { ps }\left[\Lambda_{6}^{3}\right]=\{17,18,20,36\}, \\
\text { ps }\left[\Lambda_{7}^{3}\right]=\{24,25,26,27,30,31,32,54\}, \\
\text { ps }\left[\Lambda_{8}^{3}\right]=\{33,34,35,36,37,38,39,40,41,42,44,
\end{gathered}
$$

$$
45,48,49,52,72,78,81\} \text {. }
$$

\section{A Generalization of Theorem 2 on Matrices of Class $\Lambda_{n}(\alpha, \beta, \gamma)$ with Symmetric Positions of Elements}

Denote by $\bar{\Lambda}_{n}(\alpha, \beta, \gamma)$ the set of matrices in $\Lambda_{n}(\alpha, \beta, \gamma)$ with $\beta$ 's on the main diagonal. It is clear that, together with (4),

$$
\left|\bar{\Lambda}_{n}(\alpha, \beta, \gamma)\right|=K_{n}
$$

Note that, as for sets $\Lambda_{n}^{3}, \bar{\Lambda}_{n}^{3}$, we have

$$
p s\left[\Lambda_{n}(\alpha, \beta, \gamma)\right]=p s\left[\bar{\Lambda}_{n}(\alpha, \beta, \gamma)\right] \text {. }
$$

Denote, furthermore, by $\widehat{\Lambda}_{n}(\alpha, \beta, \gamma)$ the set of matrices $M=\left\{m_{i, j}\right\}$ in $\bar{\Lambda}_{n}(\alpha, \beta, \gamma)$ with symmetric positions of elements: $m_{i, j}=\alpha$ if and only if $m_{j, i}=\gamma$. theorem.

$P$-spectrum on $\widehat{\Lambda}_{n}(\alpha, \beta, \gamma)$ is given by the following

Theorem 4. If to every partition $r \in R(n ; 3), n=n_{1}+n_{2}+\cdots+$ $n_{m}, m=m(r)$, corresponds the number

$$
H_{\alpha, \beta, \gamma}(r)=\prod_{i=1}^{m} a\left(n_{i}\right),
$$

where sequence $\{a(n)=a(\alpha, \beta, \gamma ; n\}$ is defined by the recursion

$$
\begin{gathered}
a(3)=\alpha^{3}+\beta^{3}+\gamma^{3}+3 \alpha \beta \gamma, \\
a(4)=\alpha^{4}+\beta^{4}+\gamma^{4}+4 \alpha \beta^{2} \gamma+2(\alpha \gamma)^{2}, \\
a(n)=\beta a(n-1)+\alpha \gamma a(n-2)+\alpha^{n-1}(\alpha-\beta-\gamma) \\
+\gamma^{n-1}(\gamma-\beta-\alpha), \quad n \geq 5,
\end{gathered}
$$

then one has

$$
p s\left[\widehat{\Lambda}_{n}(\alpha, \beta, \gamma)\right]=\left\{H_{\alpha, \beta, \gamma}(r): r \in R(n ; 3)\right\} .
$$

Proof. Let $S_{n}$ be the symmetric permutation group of elements $1, \ldots, n$. Two positions $\left(i_{1}, i_{2}\right),\left(j_{1}, j_{2}\right)$ are called independent if $i_{k} \neq j_{k}, k=1,2$. We will say that in the $n \times n$ matrix $M=\left\{m_{i j}\right\}$, a weight $m_{i j}$ is appropriated to the position $(i, j)$. Let $s \in S_{n}$ have no cycle of length less than $n$. Consider a map

$$
\sigma:(i, j) \longmapsto\left(s^{i}(1), s^{j}(1)\right),
$$

appropriating to the position $\left(s^{i}(1), s^{j}(1)\right)$ the weight $m_{i j}$.

Now we need several lemmas.

Lemma A. (1) The map $\sigma$ is bijective; (2) if $E$ is a set of pairwise independent positions, then $\sigma(E)$ is also a set of pairwise independent positions.

Proof. (a) Consider two distinct positions

$$
\left(i_{1}, j_{1}\right), \quad\left(i_{2}, j_{2}\right),
$$

such that, at least, one of the two inequalities holds

$$
i_{1} \neq i_{2}, \quad j_{1} \neq j_{2} .
$$

Let $i_{1} \neq i_{2}$ such that, say, $i_{1}>i_{2}$. Show that $s^{i_{1}}(1) \neq s^{i_{2}}(1)$. Indeed, if we suppose that $s^{i_{1}}(1)=s^{i_{2}}(1)$; then $s^{i_{1}-i_{2}}=1$; that is, $s$ has a cycle of length $i_{1}-i_{2}<n$ in spite of the condition. Conversely, if $s^{i_{1}}(1) \neq s^{i_{2}}(1)$, then $i_{1} \neq i_{2}$, since $s^{-1}$ has not any cycle of length less than $n$ as well.

(b) Let positions (26) be independent. Both inequalities (27) hold and, as in (a), we have $s^{i_{1}}(1) \neq s^{i_{2}}(1), s^{j_{1}}(1) \neq s^{j_{2}}(1)$; that is, the positions $\sigma\left(\left(i_{1}, j_{1}\right)\right), \sigma\left(\left(i_{1}, j_{1}\right)\right)$ are independent as well.

Lemma B. Let $s \in S_{n}$ have no cycle of length less than $n$. Then $(0,1)$-matrix $S$ having 1's on only positions

$$
\begin{aligned}
& \left(s^{1}(1), s^{2}(1)\right),\left(s^{2}(1), s^{3}(1)\right), \ldots, \\
& \left(s^{n-1}(1), s^{n}(1)\right),\left(s^{n}(1), s^{1}(1)\right)
\end{aligned}
$$

is an incidence matrix of $s$.

Proof. Since $s$ has no cycles of length less than $n$, then $\left\{s^{1}(1)\right.$, $\left.\ldots, s^{n}(1)\right\}$ is a permutation of numbers $\{1, \ldots, n\}$. Thus the set of positions of 1's of matrix $S$ coincides with the set of 1's of the incidence matrix of $s:(1, s(1)), \ldots,(n, s(n))$.

Let $P=P_{n}$ be $(0,1)$-matrix with 1's on positions $(1,2)$, $(2,3), \ldots,(n-1, n),(n, 1)$ only.

Lemma C. Let $s \in S_{n}$ have not got any cycle of length less than $n$. If $S$ and $S^{-1}$ are the incidence matrices of $s$ and $s^{-1}$, then one has

$$
\sigma^{-1}(S)=P, \quad \sigma^{-1}\left(S^{-1}\right)=P^{-1}
$$

Proof. Both formulas follow from Lemma B.

Noting that $\sigma(I)=I$, where $I$ is the identity matrix, we conclude that

$$
S^{-1}+I+S=\sigma\left(P^{-1}+I+P\right) \text {. }
$$


Moreover, since, by the bijection $\sigma$, to every diagonal (i.e., to every set of $n$ pairwise independent positions) of the matrix $\alpha S^{-1}+\beta I+\gamma S$ corresponds one and only one diagonal of the matrix $\alpha P^{-1}+\beta I+\gamma P$ with the same products of weights, then we have

$$
\operatorname{per}\left(\alpha S^{-1}+\beta I+\gamma S\right)=\operatorname{per}\left(\alpha P^{-1}+\beta I+\gamma P\right) .
$$

Note that from the definition it follows that, for every matrix $M \in \widehat{\Lambda}_{n}(\alpha, \beta, \gamma)$, we have a representation

$$
M=\alpha S^{-1}+\beta I_{n}+\gamma S,
$$

where $S$ is the incidence matrix of a substitution $s$. In case when $s$ has not got any cycle of length less than $n$, the matrix $M$ is a completely indecomposable matrix in $\widehat{\Lambda}_{n}(\alpha, \beta, \gamma)$. Thus, by (31), all completely indecomposable matrices of $\widehat{\Lambda}_{n}(\alpha, \beta, \gamma)$ have the same permanent, equal to $\operatorname{per}\left(\alpha P^{-1}+\right.$ $\left.\beta I_{n}+\gamma P\right)$.

In general, a substitution $s$ with the incidence matrix $S$ in (32) cannot have cycles of length less than 3 . Indeed, if, for some $i$, we have either $s(i)=i$ or $s(s(i))=i$, then in both cases $s(i)=s^{-1}(i)$ which means coincidence of positions 1's of the matrices $S$ and $S^{-1}$ in the $i$ th row.

Let $s \in S_{n}$ be an arbitrary substitution with cycles of length more than 2. Let

$$
s=\prod_{j=1}^{r} s_{j}
$$

where $s_{j} \in S_{l_{j}}, l_{j} \geq 3$, and $\sum_{j=1}^{r} l_{j}=n$, be the decomposition of $s$ in a product of cycles. Then the matrix $M=\alpha S^{-1}+\beta I_{n}+\gamma S$ is a direct sum of the matrices $M_{j}=\alpha S_{l_{j}}^{-1}+\beta I+\gamma S_{l_{j}}$ such that, by (31), $\operatorname{per} M_{j}=\operatorname{per}\left(\alpha P^{-1}+\beta I_{l_{j}}+\gamma P\right)$ and we have

$$
\operatorname{per} M=\prod_{j=1}^{r} \operatorname{per} M_{j}=\prod_{j=1}^{r} \operatorname{per}\left(\alpha P^{-1}+\beta I_{l_{j}}+\gamma P\right) \text {. }
$$

It is left to notice that Minc [20] found a recursion (23) for $\operatorname{per}\left(\alpha I_{n}+\beta P+\gamma P^{2}\right)$ and, as well known, the multiplication of an $n \times n$ matrix by $P^{-1}$ does not change its permanent. Therefore, $\operatorname{per}\left(\alpha P^{-1}+\beta I_{l_{j}}+\gamma P\right)=\operatorname{per}\left(\alpha I_{n}+\beta P+\gamma P^{2}\right)$.

Example 5. Let us find $p s\left[\widehat{\Lambda}_{11}(-1,3,2)\right]$.

We have the following partitions of 11 with the parts not less than 3:

$$
11=8+3=7+4=6+5=3+4+4=3+3+5 .
$$

According to (23), for $a(n)=a(-1,3,2 ; n)$, we have $a(3)=16$, $a(4)=34$ and for $n \geq 5$

$$
a(n)=3 a(n-1)-2 a(n-2)+6(-1)^{n} .
$$

Using induction, we find

$$
a(n)= \begin{cases}2^{n+1}, & \text { if } n \text { is odd } \\ 2^{n+1}+2, & \text { if } n \text { is even }\end{cases}
$$

Therefore,

$$
\begin{aligned}
\operatorname{ps}\left[\widehat{\Lambda}_{11}(-1,3,2)\right]= & \{a(11), a(3) a(8), a(5) a(6), \\
& \left.a^{2}(3) a(5), a(3) a^{2}(4)\right\} \\
= & \{4096,8224,8320,8704,16384,18496\} .
\end{aligned}
$$

In the following examples we calculate $p$-spectrum for arbitrary $n$.

Example 6. Let us find $p s\left[\widehat{\Lambda}_{n}(-1,2,1)\right]$.

By induction, for $a(n)=a(-1,2,1 ; n)$, we have

$$
a(n)= \begin{cases}2, & \text { if } n \text { is odd } \\ 4, & \text { if } n \text { is even }\end{cases}
$$

Further, again using induction, one can find that, if $n$ is even, then

$$
p s\left[\widehat{\Lambda}_{n}(-1,2,1)\right]=\left\{4,4^{2}, \ldots, 4^{\lfloor(n / 4)\rfloor}\right\}
$$

and, if $n$ is odd, then

$$
p s\left[\widehat{\Lambda}_{n}(-1,2,1)\right]=\left\{2,2 \cdot 4,2 \cdot 4^{2}, \ldots, 2 \cdot 4^{\lfloor((n-3) / 4)\rfloor}\right\} .
$$

Example 7. Analogously, in case of $\widehat{\Lambda}_{n}(-1,1,1)$, for $a(n)=$ $a(-1,1,1 ; n)$, we have

$$
\begin{gathered}
a(n)= \begin{cases}4, & \text { if } n \equiv 0(\bmod 6), \\
-2, & \text { if } n \equiv 3(\bmod 6), \\
1, & \text { otherwise, }\end{cases} \\
\text { ps }\left[\widehat{\Lambda}_{n}(-1,1,1)\right] \\
=\left\{\begin{array}{cc}
\{1,-2,4, \ldots, \\
\left.(-2)^{\lfloor(n-3) / 3\rfloor}\right\}, & \text { if } n \equiv 1,2(\bmod 3), \\
\left\{1,-2,4, \ldots,(-2)^{\lfloor(n-6) / 3\rfloor},\right. & \text { if } n \equiv 0(\bmod 3) . \\
\left.(-2)^{\lfloor n / 3\rfloor}\right\}, &
\end{array}\right.
\end{gathered}
$$

It is interesting that, in case of $n$ multiple of 3 , the permanent omits the value $(-2)^{\lfloor(n-3) / 3\rfloor}$.

\section{Merriell-Type Theorems in a Subclass of $\widehat{\Lambda}_{n}(\alpha, \beta, \gamma)$}

Note that in class $\Lambda_{n}(\alpha, \beta, \gamma)$ the Minc-Bregman inequality and the Merriell theorem, generally speaking, do not hold even for positive $\alpha, \beta$, and $\gamma$. Nevertheless, some restrictions on $\alpha, \beta$, and $\gamma$ allow proving some analogs of the Merriell theorem. Recall that $M(n)(16)$ is attained in $\widehat{\Lambda}_{n}^{3}$. Denote by $M_{n}(\alpha, \beta, \gamma)$ the maximal value of permanent in $\widehat{\Lambda}_{n}(\alpha, \beta, \gamma)$.

Theorem 8. Consider a class $\widehat{\Lambda}_{n}(\alpha, \beta, \gamma)$ with the numbers $\alpha$, $\beta$, and $\gamma$ satisfying "triangle inequalities"

$$
0 \leq \alpha \leq \beta+\gamma, \quad 0 \leq \gamma \leq \alpha+\beta,
$$


and the following additional conditions:

$$
a^{3}(4) \leq a^{4}(3), \quad \alpha \gamma+\beta(a(3))^{1 / 3} \leq(a(3))^{2 / 3},
$$

where sequence $\{a(n)\}$ is defined by recursion (23). Then, for $n$ multiple of 3 , one has

$$
M_{n}(\alpha, \beta, \gamma)=(a(3))^{n / 3} .
$$

Proof. Note that conditions (44)-(45) are satisfied, for example, in case $\alpha=\beta=\gamma=1$. Using induction, let us prove that

$$
a(n) \leq(a(3))^{n / 3} .
$$

Indeed, for $n=3$, this inequality is trivial, while, for $n=4$, it follows from the first condition (45). Let it hold for $n \leq$ $m-1$. Then, according to (23), we have

$$
\begin{aligned}
a(m)= & \beta a(m-1)+\alpha \gamma a(m-2)+\alpha^{m-1}(\alpha-\beta-\gamma) \\
& +\gamma^{m-1}(\gamma-\alpha-\beta) \\
\leq & \beta(a(3))^{(m-1) / 3}+\alpha \gamma(a(3))^{(m-2) / 3} \\
= & (a(3))^{(m-2) / 3}\left(\beta(a(3))^{1 / 3}+\alpha \gamma\right) \\
\leq & (a(3))^{(m-2) / 3}(a(3))^{2 / 3}=(a(3))^{m / 3} .
\end{aligned}
$$

Note that, according to Theorem 4 , the equality in (47) holds in a direct sum of $(3 \times 3)$-matrices of $\widehat{\Lambda}_{3}(\alpha, \beta, \gamma)$ which corresponds to the partition $n=3+3+\cdots+3$. Let now $A \in$ $\widehat{\Lambda}_{n}(\alpha, \beta, \gamma)$. By Theorem 4 , there exists a partition of $n$ with the parts not less than $3, n=n_{1}+\cdots+n_{m}$, such that

$$
\operatorname{per} A=\prod_{i=3}^{m} a\left(n_{i}\right)
$$

and, in view of (47), we have

$$
\operatorname{per} A \leq \prod_{i=3}^{m} a(3)^{n_{i} / 3}=(a(3))^{n / 3} .
$$

This proves (46).

Example 9. Consider case $\beta=\gamma-\alpha$.

Let us find the values of $\alpha$, depending on the magnitude of $\gamma$, for which the conditions of Theorem 8 are satisfied. According to (23), we have

$$
\begin{aligned}
a(3)=\alpha^{3} & +(\gamma-\alpha)^{3}+\gamma^{3}+3 \alpha(\gamma-\alpha) \gamma=2 \gamma^{3}, \\
a(4)= & \alpha^{4}+(\gamma-\alpha)^{4}+\gamma^{4}+4 \alpha(\gamma-\alpha)^{2} \gamma \\
& +2(\alpha \gamma)^{2}=2\left(\alpha^{4}+\gamma^{4}\right) .
\end{aligned}
$$

Thus the condition $a^{3}(4) \leq a^{4}(3)$ means that $\alpha^{4}+\gamma^{4} \leq$ $2^{1 / 3} \gamma^{4}$ or

$$
0 \leq \alpha \leq\left(2^{1 / 3}-1\right)^{1 / 4} \gamma=0.7140199 \cdots \gamma .
$$

And it is easy to verify that the second condition in (45) is satisfied as well. As a corollary, we obtain the following result.
Theorem 10. If (52) holds, then, for $n$ multiple of 3 , one has

$$
M_{n}(\alpha, \gamma-\alpha, \gamma)=2^{n / 3} \gamma^{n}
$$

Simple forms of sequence $\{a(n)\}$ in Examples 5 and 6 allow supposing that in case $\beta=\gamma-\alpha$ (or symmetrical case $\beta=\alpha-\gamma)$ sequence $\{a(n)\}$ keeps a sufficiently simple form. We find this form in the following lemma.

Lemma 11. If $\beta=\gamma-\alpha$, then sequence $\{a(n)\}$ which is defined by recursion (23) has the form

$$
a(n)= \begin{cases}2 \gamma^{n}, & \text { if } n \text { is odd }, \\ 2\left(\alpha^{n}+\gamma^{n}\right), & \text { if } n \text { is even. }\end{cases}
$$

Proof. Using induction with the base (51), suppose that (54) holds for $m \leq n$. Then, by (23), for even $n$, we have

$$
\begin{aligned}
a(n+1) & =(\gamma-\alpha) a(n)+\alpha \gamma a(n-1)+2 \alpha^{n}(\alpha-\gamma) \\
& =2(\gamma-\alpha)\left(\alpha^{n}+\gamma^{n}\right)+2 \alpha \gamma^{n}+2 \alpha^{n}(\alpha-\gamma)=2 \gamma^{n+1},
\end{aligned}
$$

while, if $n$ is odd, then we have

$$
\begin{aligned}
a(n+1)= & 2(\gamma-\alpha) \gamma^{n}+2 \alpha \gamma\left(\alpha^{n-1}+\gamma^{n-1}\right) \\
& +2 \alpha^{n}(\alpha-\gamma)=2\left(\alpha^{n+1}+\gamma^{n+1}\right)
\end{aligned}
$$

Let now

$$
\alpha \geq\left(2^{1 / 3}-1\right)^{1 / 4} \gamma=0.7140199 \cdots \gamma .
$$

Theorem 12. If (57) holds, then, for $n$ multiple of 4 , one has

$$
M_{n}(\alpha, \gamma-\alpha, \gamma)=\left(2\left(\alpha^{4}+\gamma^{4}\right)\right)^{n / 4} .
$$

Proof. From (51) and (57), we conclude that

$$
a(3) \leq(a(4))^{3 / 4} \text {. }
$$

Let us show that, for $n \geq 3$,

$$
a(n) \leq(a(4))^{n / 4} .
$$

For $n=4$, inequality (60) is trivial. For $n \geq 5$, we have

$$
\begin{aligned}
\alpha^{n}+\gamma^{n} & =\alpha^{n}\left(1+\left(\frac{\gamma}{\alpha}\right)^{n}\right) \\
& \leq \alpha^{n}\left(1+\left(\frac{\gamma}{\alpha}\right)^{4}\right)^{n / 4} \leq\left(\alpha^{4}+\gamma^{4}\right)^{n / 4}
\end{aligned}
$$

and thus, using Lemma 11, we have

$$
a(n) \leq 2\left(\alpha^{n}+\gamma^{n}\right)<2^{n / 4}\left(\alpha^{4}+\gamma^{4}\right)^{n / 4}=(a(4))^{n / 4}, \quad n \geq 3 .
$$


Let now $A \in \widehat{\Lambda}_{n}(\alpha, \beta, \gamma)$. By Theorem 4 , there exists a partition of $n$ with the parts not less than $3, n=n_{1}+\cdots+n_{m}$, such that

$$
\operatorname{per} A=\prod_{i=3}^{m} a\left(n_{i}\right)
$$

and, in view of (60), we have

$$
\operatorname{per} A \leq \prod_{i=3}^{m} a(4)^{n_{i} / 4}=(a(3))^{n / 4}
$$

with the equality in a direct sum of $(4 \times 4)$-matrices of $\widehat{\Lambda}_{3}(\alpha, \beta, \gamma)$ which corresponds to the partition $n=4+4+$ $\cdots+4$.

Note that, if $\alpha \neq\left(2^{1 / 3}-1\right)^{1 / 4} \gamma$, then in Theorem 10 we have only maximizing matrix (up to a permutation of the rows and columns) which corresponds to the partition $n=3+3+\cdots+$ 3 ; in Theorem 12 we also have only maximizing matrix (up to a permutation of the rows and columns) which corresponds to the partition $n=4+4+\cdots+4$. It is interesting that, only in case of the equality $\alpha=\theta \gamma$, where $\theta=\left(2^{1 / 3}-1\right)^{1 / 4}$, Theorems 10-12 are true for every $n$ multiple of 12 with the equality of the maximums $\left(2\left(\alpha^{4}+\gamma^{4}\right)\right)^{n / 4}=2^{n / 3} \gamma^{n}$. Thus, up to a positive factor $\gamma$, the class

$$
\widehat{\Lambda}_{n}(\theta, 1-\theta, 1), \quad \theta=\left(2^{1 / 3}-1\right)^{1 / 4},
$$

possesses an interesting extremal property: it contains $(n / 12)+1$ maximizing matrices (up to a permutation of the rows and columns), instead of only maximizing matrix, if $\theta \neq\left(2^{1 / 3}-1\right)^{1 / 4}$.

Indeed, the number of the maximizing matrices (up to a permutation of the rows and columns) is defined by the number of the following partitions of $n \equiv 0(\bmod 12)$ :

$$
\begin{gathered}
n=3+3+\cdots+3, \quad n=(4+4+4)+(3+\cdots+3), \ldots, \\
n=\underbrace{4+\cdots+4}_{3 i}+\underbrace{3+\cdots+3}_{n-12 i}, \quad i=0,1, \ldots, \frac{n}{12} .
\end{gathered}
$$

\section{Estimate of Cardinality of $P$-Spectrum on Circulants in $\Lambda_{n}^{3}$ and $\Lambda_{n}(\alpha, \beta, \gamma)$}

Denote by $\Delta_{n}^{3} \subset \Lambda_{n}^{3}$ the set of the circulants in $\Lambda_{n}^{3}$. Note that a circulant $A \in \Delta_{n}^{3}$ has a form $A=P^{i}+P^{j}+P^{k}, 0 \leq i<j<k \leq$ $n$, where $P=P_{n}$ is $(0,1)$-matrix with 1 's on positions $(1,2)$, $(2,3), \ldots,(n-1, n),(n, 1)$ only. Multiplying $A$ by $P^{-i}$ we obtain circulant $B$ of the form

$$
B=I_{n}+P^{r}+P^{s}
$$

with $\operatorname{per} B=\operatorname{per} A$. Since $B$ is defined by a choice of two different values $0<r<k \leq n$, then trivially

$$
p s\left[\Delta_{n}^{3}\right] \leq\left(\begin{array}{l}
n \\
2
\end{array}\right)<\frac{n^{2}}{2} .
$$

Now we prove essentially more exact and practically unimprovable estimate.
Theorem 13. One has

$$
p s\left[\Delta_{n}^{3}\right] \leq\left\lfloor\frac{n^{2}+3}{12}\right\rfloor .
$$

Proof. Let us return back to the general form

$$
A=P^{i}+P^{j}+P^{k}, \quad 0 \leq i<j<k \leq n .
$$

Note that $A$ is defined by a choice of a vector $(i, j, k)$, but its rotation, that is, a passage to a vector of the form $(i+l, j+l, k+l)(\bmod n)$, does not change the magnitude of per $A$. Indeed it corresponds to the multiplication $A$ by $P^{l}$, and our statement follows from the equality $\operatorname{per}\left(P^{l} A\right)=$ $\operatorname{per} A$. Besides, its reflection relative to some diameter of the imaginary circumference of the rotation, by the symmetry, keeps magnitude of the permanent. Since geometrically three points on the imaginary circumference define a triangle, then our problem reduces to a triangle case of the following general problem, posed by Professor Richard H. Reis (South-East University of Massachusetts, USA) in a private communication to Hansraj Gupta in 1978:

Let a circumference is split by the same $n$ parts. It is required to find the number $R(n, k)$ of the incongruent convex $k$-gons, which could be obtained by connection of some $k$ from $n$ dividing points. Two $k$-gons are considered congruent if they are coincided at the rotation of one relatively other along the circumference and (or) by reflection of one of the $k$-gons relatively some diameter.

In 1979, Gupta [21] gave a solution of the Reis problem in the form

$$
\left.\begin{array}{rl}
R(n, k)=\frac{1}{2}( & \left(\begin{array}{c}
\left\lfloor\left(\frac{n-h_{k}}{2}\right)\right\rfloor \\
\left\lfloor\left(\frac{k}{2}\right)\right\rfloor
\end{array}\right)+\frac{1}{k} \\
& \times \sum_{d \mid \operatorname{gcd}(k, n)} \varphi(d) \frac{\frac{n}{d}-1}{d}-1
\end{array}\right) .
$$

If we denote by $\Delta_{n}^{k} \subset \Lambda_{n}^{k}$ the set of the circulants in $\Lambda_{n}^{k}$, then from our arguments it follows that

$$
p s\left[\Delta_{n}^{k}\right] \leq R(n, k) \text {. }
$$

In case $k=3$, from (71)-(72) we find

$$
p s\left[\Delta_{n}^{3}\right] \leq \begin{cases}\frac{n^{2}}{12}, & \text { if } n \equiv 0(\bmod 6), \\ \frac{n^{2}-1}{12}, & \text { if } n \equiv 1,5(\bmod 6), \\ \frac{n^{2}-4}{12}, & \text { if } n \equiv 2,4(\bmod 6), \\ \frac{n^{2}+3}{12}, & \text { if } n \equiv 3(\bmod 6),\end{cases}
$$

and (69) follows. 
Example 14. In case $n=5$ we have only two incongruent triangles corresponding to circulants $I_{5}+P+P^{2}$ and $I_{5}+P+P^{3}$.

Nevertheless, the calculations give $\operatorname{per}\left(I_{5}+P+P^{2}\right)=$ $\operatorname{per}\left(I_{5}+P+P^{3}\right)=13$. Thus $p s\left[\Delta_{5}^{3}\right]=\{13\}$, and $\left|p s\left[\Delta_{5}^{3}\right]\right|=1$.

Example 15. In case $n=6$ we have three incongruent triangles corresponding to circulants $I_{6}+P+P^{2}, I_{6}+P+P^{3}$, and $I_{6}+P^{2}+P^{4}$.

The calculations give $\operatorname{per}\left(I_{6}+P+P^{2}\right)=20$ and $\operatorname{per}\left(I_{6}+\right.$ $\left.P+P^{3}\right)=17$, while $\operatorname{per}\left(I_{6}+P^{2}+P^{4}\right)=36$. Thus $p s\left[\Delta_{6}^{3}\right]=$ $\{17,20,36\}$, and $\left|p s\left[\Delta_{6}^{3}\right]\right|=3$.

Note that a respectively large magnitude of $\operatorname{per}\left(I_{6}+P^{2}+\right.$ $\left.P^{4}\right)$ is explained by its decomposability in a direct product of circulants $\left(I_{3}+P+P^{2}\right) \otimes\left(I_{3}+P+P^{2}\right)$, such that $\operatorname{per}\left(I_{6}+P^{2}+P^{4}\right)=$ $\left(\operatorname{per}\left(I_{3}+P+P^{2}\right)\right)^{2}=6^{2}=36$.

It is clear that, in case of circulants in $\Lambda_{n}(\alpha, \beta, \gamma)$, the upper estimate (69) yields either the same estimate, if $\alpha=\beta=$ $\gamma$, or $\left\lfloor\left(n^{2}+3\right) / 4\right\rfloor$, if $\alpha=\beta \neq \gamma$ (and in the symmetric cases), or $\left\lfloor\left(n^{2}+3\right) / 2\right\rfloor$, if $\alpha, \beta$, and $\gamma$ are distinct numbers.

Add that, using a bijection, one can apply formula (71) to enumerate the two-color bracelets of $n$ beads, $k$ of which are black and $n-k$ are white (see, e.g., the author's explicit formulas for sequences A032279-A032282, A005513-A005516 in $[15])$.

\section{Algorithm of Calculations of Upper Magnitudes of the Permanent in $\widehat{\Lambda}_{n}^{3}$}

Theorem 2 allows, using some additional arguments, giving an algorithm of explicit expressions of upper magnitudes of the permanent in $\widehat{\Lambda}_{n}^{3}$ via numbers (14). For that we need the following lemma.

Lemma 16. For $n_{1}, n_{2} \geq 3$, one has

$$
a\left(n_{1}+n_{2}\right) \leq a\left(n_{1}\right) a\left(n_{2}\right), \quad n_{1}, n_{2} \geq 3 .
$$

Proof. By the usual way, from (14) we find

$$
a(n)=\varphi^{n}+2+(-1)^{n} \varphi^{-n},
$$

where $\varphi=(\sqrt{5}+1) / 2$ is the golden ratio.

Denote $\varepsilon(n)=(-1)^{n} \varphi^{-n}$. Since $n \geq 3$, then $|\varepsilon(n)|<0.24$, and, consequently, if $n=n_{1}+n_{2}$, then $\left(2+\varepsilon\left(n_{1}\right)\right)\left(2+\varepsilon\left(n_{2}\right)\right)>$ $1.76^{2}>3$. Therefore, we have

$$
\begin{aligned}
a\left(n_{1}\right) a\left(n_{2}\right) & =\left(\varphi^{n_{1}}+2+\varepsilon\left(n_{1}\right)\right)\left(\varphi^{n_{2}}+2+\varepsilon\left(n_{2}\right)\right) \\
& >\varphi^{n_{1}+n_{2}}+3>\varphi^{n_{1}+n_{2}}+2+\varepsilon\left(n_{1}+n_{2}\right) \\
& =a\left(n_{1}+n_{2}\right) .
\end{aligned}
$$

Note that, actually, the difference between the hand sides of (76) is more than $1.76\left(\varphi^{n_{1}}+\varphi^{n_{2}}\right)$.

Let now $n \equiv j(\bmod 3), j=0,1$, and 2 , and $t \in \mathbf{N}$. Let $R(m ; v)$ denote the set of all partitions of $n$ with parts more than or equal to $\nu$. For us an important role play cases $\nu=3,4$. To $r \in R(m ; 3), \rho \in R(m ; 4)$ put in a correspondence the sets

$$
H_{m ; 3}(r)=\left\{\Pi_{r_{i} \in r} a_{r_{i}}\right\}, \quad H_{m ; 4}(\rho)=\left\{\Pi_{\rho_{i} \in \rho} a_{\rho_{i}}\right\} .
$$

In case $m=3$, when $\rho=\emptyset$, let us agree that $H_{3 ; 4}$ is a singleton $\{6\}$.

Consider now the set $L_{t}^{(j)}=L_{t}^{(j)}(n)$ :

$$
L_{t}^{(j)}=\bigcup_{i=1}^{4 t+j}\left\{6^{(n-j-3 i) / 3} y: y \in H_{3 i+j ; 4}(\rho), y \geq 9^{3 t+j} 6^{i-4 t-j}\right\} .
$$

Theorem 17 (algorithm of calculation of upper magnitudes of the permanent in $\left.\widehat{\Lambda}_{n}^{3}\right)$. If $n \geq 4(3 t+j)$, then the set $L_{t}^{(j)}$ taken in decreasing order of its elements gives $\left|L_{t}^{(j)}\right|$ upper magnitudes of the permanent in $\widehat{\Lambda}_{n}^{3}$.

Proof. Note that the proof is the same for every value of $j$. Therefore, let us consider, say, $j=0$. From (74) it follows that, if $r \in R(n ; 3)$ contains $\lambda_{3}$ parts 3 and $\lambda_{3} \leq(n / 3)-4 t$, then, for $y \in H_{n-3 \lambda_{3} ; 4}(\rho)$, we have

$$
6^{\lambda_{3}} y \leq 6^{(n / 3)-4 t} 9^{3 t} .
$$

This means that, for the formation of the list of all upper magnitudes of the permanent in $\widehat{\Lambda}_{n}^{3}$ in the condition $n \geq 12 t$, which are bounded from below by $6^{(n / 3)-4 t} 9^{3 t}$, it is sufficient to consider only a part of the spectrum containing numbers $\left\{6^{\lambda_{3}} y\right\}$, where $y \in H_{n-3 \lambda_{3} ; 4}(\rho)$ with the opposite condition $\lambda_{3} \geq(n / 3)-4 t$. From the equality $3 \lambda_{3}+\cdots+n \lambda_{n}=n$ with the condition $\lambda_{3} \geq(n / 3)-4 t$, we have

$$
4 \lambda_{4}+\cdots+n \lambda_{n} \leq n-3\left(\frac{n}{3}-4 t\right)=12 t .
$$

Since $12 t$ does not depend on $n$, there is only a finite assembly of such partition for arbitrary $n$. This ensures a possibility of the realization of the algorithm.

For the considered $r \in R(n ; 3)$, for $\lambda_{3} \geq(n / 3)-4 t$, we have $H_{n ; 3}(r)=6^{(n-m) / 3}$, where $y \in H_{m ; 4}(\rho)$, and $m$ has the form $m=3 i, 1 \leq i \leq 4 t$. Thus we should choose only $H_{n ; 3}(r) \geq$ $6^{(n / 3)-4 t} 9^{3 t}$, and this yields

$$
y \geq 9^{3 t} 6^{(n / 3)-4 t}=9^{3 t} 6^{i-4 t}, \quad i=1,2, \ldots, 4 t .
$$

In order to use Theorem 17 for calculating the upper magnitudes $M^{(1)}(n)>M^{(2)}(n)>\cdots$ of the permanent in $\widehat{\Lambda}_{n}^{3}$, in case, say, $n \equiv 0(\bmod 3)$,

(1) we write a list of partition of numbers $3 i, i=2,3, \ldots$, $4 t$, with the parts not less than 4 ;

(2) we compare the corresponding values of $y$ with $9^{3 t} 6^{i-4 t}$ and keep only $y \geq 9^{3 t} 6^{i-4 t}$

(3) after that, we take in decreasing order the numbers $\left\{y 6^{(n / 3)-i}\right\}$. 
Below we give the first 10 upper magnitudes $\widehat{M}^{(1)}>$ $\widehat{M}^{(2)}>\cdots>\widehat{M}^{(10)}$ of the permanent in $\widehat{\Lambda}_{n}^{3}$, for $n \geq 24$, via numbers $\{a(n)\}(14)$. Consider

$$
\widehat{M}^{(1)}=\left\{\begin{array}{cc}
a(3)^{n / 3}=6^{n / 3}, & \text { if } n \equiv 0(\bmod 3), \\
a(4) a(3)^{(n-4) / 3} & \\
=\frac{3}{2} 6^{(n-1) / 3}, & \text { if } n \equiv 1(\bmod 3), \\
a(4)^{2} a(3)^{(n-8) / 3} & \\
=\frac{9}{4} 6^{(n-2) / 3}, & \text { if } n \equiv 2(\bmod 3) .
\end{array}\right.
$$

Formula (82) shows that $M^{(1)}(n)$ is attained in $\widehat{\Lambda}_{n}^{3}$. Moreover

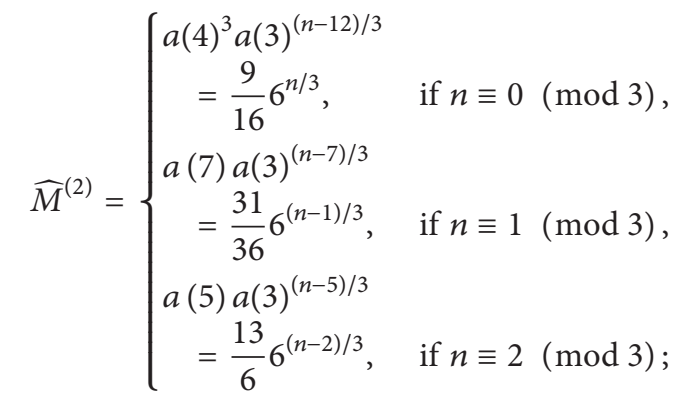

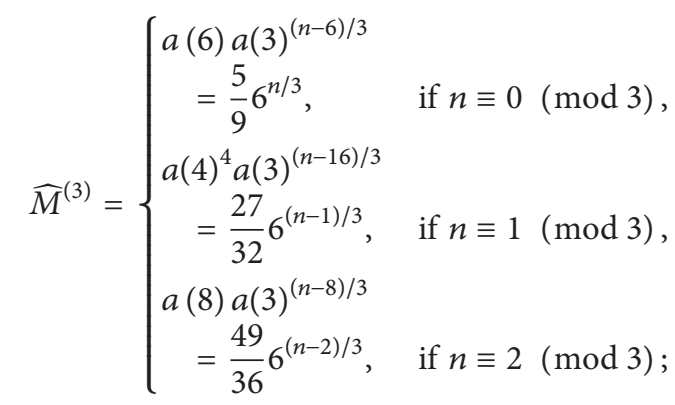

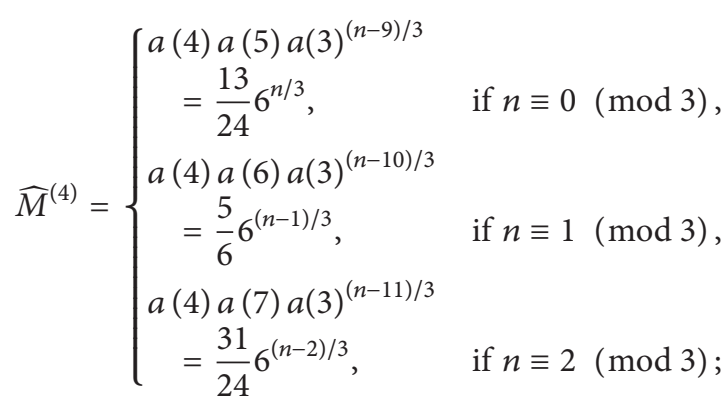

$$
\begin{aligned}
& \widehat{M}^{(5)}=\left\{\begin{aligned}
a(9) a(3)^{(n-9) / 3} & \\
=\frac{13}{36} 6^{n / 3}, & \text { if } n \equiv 0(\bmod 3), \\
a(4)^{2} a(5) a(3)^{(n-13) / 3} & \\
=\frac{13}{16} 6^{(n-1) / 3}, & \text { if } n \equiv 1(\bmod 3), \\
a(4)^{5} a(3)^{(n-20) / 3} & \\
=\frac{81}{64} 6^{(n-2) / 3}, & \text { if } n \equiv 2(\bmod 3) ;
\end{aligned}\right.
\end{aligned}
$$

\section{Main Conjectural Inequality for Maximum of Permanent in Completely Indecomposable $\Lambda_{n}^{3}$-Matrices}

Denote by $\Lambda_{n, 1}^{3}$ the set of completely indecomposable matrices in $\Lambda_{n}^{3}$, that is, the set of $\Lambda_{n}^{3}$-matrices containing no $\Lambda_{m}^{3}$ submatrices. Let $\mu_{1}(n)$ denote the maximum of permanent 
in $\Lambda_{n, 1}^{3}$. Our very plausible conjecture which we call "main conjectural inequality (MCI)" is the following.

Conjecture 18 (cf. Shevelev [11], pp. 165-166). For $n_{1}, n_{2} \geq 3$, one has

$$
\mu_{1}\left(n_{1}+n_{2}\right) \leq \mu_{1}\left(n_{1}\right) \mu_{1}\left(n_{2}\right) .
$$

In Lemma 16 we essentially proved that in subclass $\widehat{\Lambda}_{n}^{3}$ the MCI is valid.

Besides, in all known cases MCI holds. Moreover, as we shall see, our algorithm of calculation of the consecutive upper magnitudes $\left(M=M_{1}>M_{2}>\cdots\right)$ of permanent in $\Lambda_{n}^{3}$, which is based on MCI, reproduces all Merriell's and Bolshakov's results for $M_{1}$ and $M_{2}$. Note also that, for sufficiently large $n$, the number of consecutive upper magnitudes of permanent in $\Lambda_{n}^{3}$ grows very quickly with every step of extension of the list of known $p$-spectrums for small $n$. For example, using the found by Bolshakov $p s\left[\Lambda_{i}^{3}\right], i \leq 8$, we obtain, for sufficiently large $n, 4,7$, and 11 upper values of $p s\left[\Lambda_{n}^{3}\right]$ in cases $n=3 k, 3 k+1$, and $3 k+2$ respectively. Further, after calculating $p s\left[\Lambda_{9}^{3}\right]$, the number of upper values of, for example, $p s\left[\Lambda_{3 k}^{3}\right]$ increases by more than thrice.

\section{Algorithm of Calculations of Upper Magnitudes of the Permanent in $\Lambda_{n}^{3}$ Based on MCI}

Let $n \equiv j(\bmod 3), j=0,1,2$, and $t \in \mathbf{N}$. Let $R(m ; \nu)$ denote the set of all partitions of $n$ with parts more than or equal to $\nu$. For us an important role play cases $v=3,4$. To $r \in R(m ; 3)$, $\rho \in R(m ; 4)$ put in a correspondence sets

$$
\pi_{m ; 3}(r)=\left\{\Pi_{r_{i} \in r} x_{r_{i}}\right\}, \quad \pi_{m ; 4}(\rho)=\left\{\Pi_{\rho_{i} \in \rho} x_{\rho_{i}}\right\},
$$

where $x_{s}$ runs through all values of permanent in set $\Lambda_{s, 1}^{3}$ of completely indecomposable matrices in $\Lambda_{s}^{3}$ (in case $m=3$, when $\rho=\emptyset$, let us agree that $\pi_{3 ; 4}$ is a singleton $\{6\}$ ).

Consider now the set $E_{t}^{(j)}=E_{t}^{(j)}(n)$ :

$$
E_{t}^{(j)}=\bigcup_{i=1}^{4 t+j}\left\{6^{(n-j-3 i) / 3} y: y \in \pi_{3 i+j ; 4}(\rho), y \geq 9^{3 t+j} 6^{i-4 t-j}\right\} .
$$

Theorem 19 (algorithm of calculation of upper magnitudes of the permanent in $\left.\Lambda_{n}^{3}\right)$. If $n \geq 4(3 t+j)$, then the set $E_{t}^{(j)}$ taken in decreasing order of its elements gives $\left|E_{t}^{(j)}\right|$ upper magnitudes of the permanent in $\Lambda_{n}^{3}$.

Proof. We need three lemmas.

Lemma 20. For $n \geq 4$, one has

$$
\mu_{1}(n) \leq 3^{n / 2} .
$$

Proof. Let, firstly, $n \equiv 0(\bmod 4)$. Note that $\mu_{1}(4)=D_{4}=9$. Using (92), we find

$$
\mu_{1}(n) \leq \mu_{1}(4) \mu_{1}(n-4) \leq \cdots \leq\left(\mu_{1}(4)\right)^{n / 4}=3^{n / 2} .
$$

Let, furthermore, $n \equiv i(\bmod 4), i=1,2,3$. Note that, by (19), $\mu_{1}(5) \leq 13<3^{2.5}$. Therefore, using (92), we have

$$
\mu_{1}(n) \leq\left(\mu_{1}(4)\right)^{(n-5 i) / 4}\left(\mu_{1}(5)\right)^{i}<3^{(n-5 i) / 2} 3^{2.5 i}=3^{n / 2} .
$$

Lemma 21. Let $n=3 \lambda_{3}+4 \lambda_{4}+\cdots+n \lambda_{n}$ be a partition of $n$ with the parts not less than 3. If $\lambda_{3} \leq l$ and $n$ has the form $n=$ $3 l+4 m$, then, for completely indecomposable matrices $A_{i} \in \Lambda_{i}^{3}$, $i=3,4, \ldots, n$, one has

$$
\prod_{i=3}^{n}\left(\operatorname{per} A_{i}\right)^{\lambda_{i}} \leq 69^{m}
$$

Proof. Using Lemma 20, we have

$$
\prod_{i=3}^{n}\left(\operatorname{per} A_{i}\right)^{\lambda_{i}} \leq 6^{\lambda_{3}} \sqrt{3}^{4 \lambda_{4}+\cdots+n \lambda_{n}} \leq 6^{l} \sqrt{3}^{n-\lambda_{3}}=6 \sqrt{3}^{4 m}=6^{l} 9^{m} .
$$

Lemma 22. Let $n=3 \lambda_{3}+4 \lambda_{4}+\cdots+n \lambda_{n}$ and

$$
\lambda_{3} \leq \frac{n-4 j}{3}-4 t, \quad n \geq 4(3 t+j)
$$

where $t$ is a nonnegative integer and $j$ is the residue of $n$ modulo $3, j=0,1,2$; then, for completely indecomposable matrices $A_{i} \in \Lambda_{i}^{3}, i=3,4, \ldots, n$, one has

$$
\prod_{i=3}^{n}\left(\operatorname{per} A_{i}\right)^{\lambda_{i}} \leq 6^{((n-4 j) / 3)-4 t} 9^{3 t+j} .
$$

Proof. Put $l=((n-4 j) / 3)-4 t, m=(n-3 l) / 4=3 t+j$. Now the lemma follows from Lemma 21.

It is left to note that, after these lemmas, the proof of Theorem 19 is the same as proof of Theorem 17.

Note that the use of this algorithm is based on the upper values of $p$-spectrum for smaller values of $n$.

Consider, for example, case $t=0, j=2$. According to (94), we have

$$
\begin{aligned}
E_{0}^{(2)} & =\bigcup_{i=1}^{2}\left\{6^{(n-2-3 i) / 3} y: y \in \pi_{3 i+j ; 4}(\rho), y \geq 81 \cdot 6^{i-2}\right\} \\
& =\left\{6^{(n-5) / 3} \operatorname{per} A, A \in \Lambda_{5}^{3}: 6 \operatorname{per} A \geq 81\right\} \cup\left\{81 \cdot 6^{(n-8) / 3}\right\} .
\end{aligned}
$$

Note that the second set is a simpleton, since, by MCI, $\mu_{1}(8,3) \leq\left(\mu_{1}(4,3)\right)^{2}=81$. Since, by $(19), M(5)=13<81 / 6$, then the first set in (102) is empty. Thus $E_{0}^{(2)}=E_{0}^{(2)}(n)$ is simpleton:

$$
E_{0}^{(2)}=\left\{81 \cdot 6^{(n-8) / 3}\right\}
$$

and we have

$$
M^{(1)}(n)=81 \cdot 6^{(n-8) / 3}, \quad n \geq 8,
$$

which corresponds to Merriell's result in case $n \equiv 2(\bmod 3)$. 
Further research of the set (94), using (19), gives the following results.

(1) Consider $j=0, n \geq 24$ :

$$
\begin{array}{rlrl}
M^{(1)}(n) & =6^{n / 3}, & M^{(2)}(n) & =\frac{9}{16} 6^{n / 3}, \\
M^{(3)}(n)=\frac{5}{9} 6^{n / 3}, & M^{(4)}(n) & =\frac{13}{24} 6^{n / 3} .
\end{array}
$$

The continuation of this list requires knowing $p s\left[\Lambda_{9}^{3}\right]$. Note that a more detailed analysis shows that, after calculation of $p s\left[\Lambda_{9}^{3}\right]$, in this case one can obtain the first $12+|G|$ upper magnitudes of the permanent in $\Lambda_{n}^{3}$, where $G=$ $\operatorname{ps}\left[\Lambda_{9}^{3}\right] \cap([69,116] \backslash\{72,78,102,108\})$.

(2) Consider $j=1, n \geq 28$ :

$$
\begin{array}{rlrl}
M^{(1)}(n) & =\frac{3}{2} 6^{(n-1) / 3}, & M^{(2)}(n) & =\frac{8}{9} 6^{(n-1) / 3}, \\
M^{(3)}(n) & =\frac{31}{36} 6^{(n-1) / 3}, & M^{(4)}(n) & =\frac{27}{32} 6^{(n-1) / 3}, \\
M^{(5)}(n) & =\frac{5}{6} 6^{(n-1) / 3}, & M^{(6)}(n) & =\frac{13}{15} 6^{(n-1) / 3}, \\
M^{(7)}(n) & =\frac{169}{216} 6^{(n-1) / 3} . &
\end{array}
$$

It is interesting that in this case $p s\left[\Lambda_{9}^{3}\right]$ is not used up to $M^{(7)}$, but the continuation of this list requires knowing $p s\left[\Lambda_{10}^{3}\right]$.

(3) Consider $j=2, n \geq 32$ :

$$
\begin{array}{rlrl}
M^{(1)}(n) & =\frac{9}{4} 6^{(n-2) / 3}, & M^{(2)}(n) & =\frac{13}{6} 6^{(n-2) / 3}, \\
M^{(3)}(n) & =2 \cdot 6^{(n-2) / 3}, & M^{(4)}(n) & =\frac{13}{9} 6^{(n-2) / 3} \\
M^{(5)}(n) & =\frac{49}{36} 6^{(n-2) / 3}, & M^{(6)}(n) & =\frac{4}{3} 6^{(n-2) / 3}, \\
M^{(7)}(n) & =\frac{31}{24} 6^{(n-2) / 3}, & M^{(8)}(n) & =\frac{81}{64} 6^{(n-2) / 3} \\
M^{(9)}(n) & =\frac{5}{4} 6^{(n-2) / 3}, & M^{(10)}(n) & =\frac{11}{9} 6^{(n-2) / 3} \\
M^{(11)}(n) & =\frac{39}{32} 6^{(n-1) / 3} . &
\end{array}
$$

Note that the method not only gives a possibility to calculate the upper magnitudes of the permanent in $\Lambda_{n}^{3}$, but also indicates those direct products on which they are attained. For example, in (107) $M_{9}$ is attained on direct products of some matrices $A_{i} \in \Lambda_{i}^{3}$ :

$$
\begin{aligned}
& A_{8} \otimes \underbrace{A_{3} \otimes \cdots \otimes A_{3}}_{(n-8) / 3}, \quad A_{4} \otimes A_{7} \otimes \underbrace{A_{3} \otimes \cdots \otimes A_{3}}_{(n-11) / 3}, \\
& A_{4} \otimes A_{4} \otimes A_{6} \otimes \underbrace{A_{3} \otimes \cdots \otimes A_{3}}_{(n-14) / 3} .
\end{aligned}
$$

Note also that the comparison of (105)-(107) with (82)(91) shows that the following calculated $M^{(i)}$ are attained in $\widehat{\Lambda}_{n}^{3}, n \geq 32$ :

in case $n \equiv 0 \bmod 3$,

$$
M^{(1)}, M^{(2)}, M^{(3)}, M^{(4)}
$$

in case $n \equiv 1 \bmod 3$,

$$
M^{(1)}, M^{(3)}, M^{(4)}, M^{(5)}, M^{(6)}, M^{(7)}
$$

(but $M^{(2)}$ is not attained);

in case $n \equiv 2 \bmod 3$,

$$
M^{(1)}, M^{(2)}, M^{(5)}, M^{(7)}, M^{(8)}, M^{(9)}, M^{(11)}
$$

(but $M^{(3)}, M^{(4)}, M^{(6)}$, and $M^{(10)}$ are not attained).

\section{Algorithm for Testing the Parity of Values of the Permanent in $\Lambda_{n}^{3}$}

It seems that, among all known methods of calculation of the permanent, only Ryser's method (cf. [1], chapter 7) could be used for creating an algorithm for testing the parity of values of the permanent. Let $A$ be $n \times n$-matrix. Let $A_{r}$ be a matrix which is obtained by changing some $r$ columns of $A$ by zero columns. Denote by $S\left(A_{r}\right)$ the product of row sums of $A_{r}$. Then, by Ryser's formula, we have

$$
\begin{aligned}
\operatorname{per} A= & \sum S\left(A_{0}\right)-\sum S\left(A_{1}\right)+\sum S\left(A_{2}\right)-\cdots \\
& +(-1)^{n-1} \sum S\left(A_{n-1}\right) .
\end{aligned}
$$

Let now $A$ have integer elements. Introduce the following matrix function:

$$
\Upsilon(A)= \begin{cases}1, & \text { if all row sums of } A \text { are odd, } \\ 0, & \text { otherwise. }\end{cases}
$$

From (112) we have

$$
\begin{aligned}
\operatorname{per} A \equiv & \sum \Upsilon\left(A_{0}\right)-\sum \Upsilon\left(A_{1}\right)+\sum \Upsilon\left(A_{2}\right)+\cdots \\
& +\sum \Upsilon\left(A_{n-1}\right)(\bmod 2) .
\end{aligned}
$$

Using (114), let us create an algorithm of a search of the odd values of the permanent in $\Lambda_{n}^{3}$. Since, evidently, $\operatorname{per} A \equiv \operatorname{det} A(\bmod 2)$, then $A$ should have pairwise distinct columns. Note that cases $n \equiv j(\bmod 3), j=0,1,2$, are considered by the same way. Suppose, say, $n=3 t$. According to (114), we are interested in only cases when, after removing $r \geq 1$ columns of $A$, all row sums will be odd. Suppose that, after removing $r$ columns of $A$, we have that $p$ sums remain equal to 3 and $n-p$ sums equal to 1 . This means that the total number of the removed l's equals $2(n-p)=6 t-2 p$. Since, removing a column, we remove three 1's, then the number of the removed columns equals $r=2 t-((2 p) / 3)$. Thus $p=3 m$ and $r=2(t-m), m=0,1, \ldots, t-1$. However, if $m=t-1$, then $r=2$. By the condition, these two columns are distinct; therefore, we conclude that at least one row sum equals 2 . The contradiction shows that the testing sequence is $r=4,6, \ldots, 2 t$. In cases $n \equiv 1,2(\bmod 3)$ we obtain the same testing sequence. 
Example 23. Let us check the parities of values of the permanent of circulants in $\Delta_{7}^{3} \subset \Lambda_{7}^{3}$.

In this case $t=\lfloor 7 / 3\rfloor=2$ and, therefore, the testing sequence contains only term $r=4$. Note that matrix $A_{r}$ has all odd rows if and only if one row sum equals 3 and each of the 6 other row sums equals 1 . Indeed, let, after removing 4 columns of $A, p$ sums remain equal to 3 and $7-p$ sums equal to 1 . This means that the total number of the removed l's equals $2(7-p)$ and the number of the removed columns equals $r=4=(14-2 p) / 3$; that is, $p=1$. Moreover, since in a circulant all rows are congruent shifts of the first one, it is sufficient to consider the case when precisely the first row sum equals 3 and others equal 1 (the multiplication on 7 does not change the parity of the result). This opens a possibility of a momentary handy test on the parity every circulant of class $\Delta_{7}^{3}$. This test consists of removing all four columns beginning with 0 . If now every row $2, \ldots, 7$ has one 1 , then the permanent is even; otherwise, it is odd. We check now directly that from $\left(\begin{array}{l}7 \\ 3\end{array}\right)=35$ circulants exactly 21 ones have odd permanent.

Remark 24. In 1967, Ryser [9] did a conjecture that the number of the transversals of a Latin square from elements $1, \ldots, n$ (i.e., the number of subsets of its $n$ pairwise distinct elements, with none in the same row or column) has the same parity as $n$. If $n$ is even, then the conjecture has been proved by Balasubramanian [8]. Besides, in [8] Balasubramanian posed a conjecture for the parity of a sum of permanents, such that the truth of this conjecture yields Ryser's hypothesis for odd $n$. In the same year (1990), using the result of Example 23, the author disproved Balasubramanian's conjecture. It is interesting that soon Parker (see [22], p. 258) indeed found several Latin squares of order 7 with even number of transversals. Add that later ([7]) we found even an infinite set of counterexamples to the Balasubramanian conjecture.

\section{Open Problems}

(1) To prove the MCI (Section 7).

(2) Consider class $\Lambda_{n}(1,1+a, 1+b)$, where $0 \leq a \leq b<$ $(4 e-9) / 6$ (cf. [23], pp. 171-172). Since $\Lambda_{n}(1,1,1)=\Lambda_{n}^{3}$, then Voorhoeve's lower estimate $(81 / 32)(4 / 3)^{n}$ for permanent of $\Lambda_{n}^{3}$-matrices trivially holds for matrices in $\Lambda_{n}(1,1+a, 1+b)$. It is clear that, for $a>0$, $b>0$, an essentially stronger lower estimate should exist. However, using van der Waerden-EgorychevFalikman theorem to class $\Lambda_{n}(1 /(3+a+b),(1+a) /(3+$ $a+b),(1+b) /(3+a+b))$ of doubly stochastic matrices, for the permanent of $\Lambda_{n}(1,1+a, 1+b)$-matrices, we obtain even weaker lower estimate of the order $C_{1} \sqrt{n}((3+a+b) / e)^{n} \ll C(4 / 3)^{n}$ in the case $a+b \epsilon$ $(0,(4 e-9) / 3)$. The problem is to find a stronger lower estimate for the permanent in $\Lambda_{n}(1,1+a, 1+b)$ in this case.

(3) Let $M$ be a circulant of order $n$ with integer elements (cf. [23], pp. 115-116). We conjecture that, for every integer $m$, we have per $M \equiv(-1)^{n} \operatorname{per}\left(m J_{n}-M\right)(\bmod$ $n$ ), where $n \times n$-matrix $J_{n}$ consists of 1's only. In [7] the conjecture was proved for every $m$ and prime $n$. Thus the question is open in case of arbitrary composite number $n$.

(4) Let us call two Latin rectangles equivalent if the sets of their elements in the corresponding columns are the same. Note that one can treat numbers $\left|\Lambda_{n}^{3}\right|$ as the numbers of equivalence classes of $3 \times n$ Latin triangles. Let $A=I_{n}+P+P^{2}$. It could be proved that the cardinality of the corresponding equivalent class is $2^{n}+6+2(-1)^{n}$. To find the cardinality of the equivalent class which is defined by matrix $I_{n}+P+P^{3}$.

\section{Acknowledgment}

The author is grateful to both of the anonymous referees for several suggestions which promoted improvement in the text.

\section{References}

[1] H. Minc, Permanents, Addison-Wesley, 1978.

[2] G. P. Egorychev, “The solution of van der Waerden's problem for permanents," Advances in Mathematics, vol. 42, no. 3, pp. 299305, 1981.

[3] D. I. Falikman, "Proof of the van der Waerden's conjecture on the permanent of a doubly stochastic matrix," Matematicheskie Zametki, vol. 29, no. 6, pp. 931-938, 1981 (Russian).

[4] L. M. Brègman, "Certain properties of nonnegative matrices and their permanents," Doklady Akademii Nauk SSSR, vol. 211, pp. 27-30, 1973.

[5] N. Alon and S. Friedland, "The maximum number of perfect matchings in graphs with a given degree sequence," Electronic Journal of Combinatorics, vol. 15, no. 1, article13, 2 pages, 2008.

[6] D. Merriell, “The maximum permanent in $\Lambda_{n}^{k}$ ”, Linear and Multilinear Algebra, vol. 9, no. 2, pp. 81-91, 1980.

[7] V. S. Shevelev, "An algorithm for testing the parity of a permanent (or determinant) and counterexamples to a conjecture of K. Balasubramanian," VINITI, no. 1692-B91, Moscow, Russia, 1991 (Russian).

[8] K. Balasubramanian, "On transversals in latin squares," Linear Algebra and Its Applications, vol. 131, pp. 125-129, 1990.

[9] H. Ryser, "Neuere probleme in der kombinatorik," in Vortrage uber Komb, pp. 69-91, Oberwolfash, 1967.

[10] V. E. Tarakanov, "Combinatorial problems on binary matrices," in Combinatorial Analysis, vol. 5, pp. 4-15, MSU, Moscow, Russia, 1980 (Russian).

[11] V. S. Shevelev, "Some problems in the theory of enumeration of permutations with restricted positions," in Itogi Nauki i Tekhniki, Seriya Teoriya Veroyatnostei, Matematicheskaya Statistika, Teoreticheskaya Kibernetika, vol. 30, pp. 113-177, 1992 (Russian).

[12] R. Stanley, Enumerative Combinatorics, vol. 1, Wadsworth, Belmont, Calif, USA, 1986.

[13] P. E. O’Neil, "Asymptotics and random matrices with row-sum and column-sum restrictions," Bulletin of the American Mathematical Society, vol. 75, pp. 1276-1282, 1969.

[14] J. Riordan, An Introduction to Combinatorial Analysis, John Wiley \& Sons, New York, NY, USA, 1967. 
[15] N. J. A. Sloane, "The On-Line Encyclopedia of Integer Sequences," http://oeis.org/.

[16] V. S. Shevelev, "Reduced Latin rectangles and square matrices with identical sums in the rows and columns," Diskretnaya Matematika, vol. 4, no. 1, pp. 91-110, 1992.

[17] V. I. Bolshakov, "On upper values of a permanent in $\Lambda_{n}^{k}$ " in Combinatorial Analysis, vol. 7, pp. 92-118, MSU, Moscow, Russia, 1986 (Russian).

[18] M. Voorhoeve, "A lower bound for the permanents of certain (0,1)-matrices,' Indagationes Mathematicae, vol. 41, no. 1, pp. 83-86, 1979.

[19] V. I. Bolshakov, "The spectrum of the permanent on $\Lambda_{n}^{k}$ ”, in Proceedings of the All-Union seminar on discrete mathematics and its applications, pp. 65-73, MSU, Moscow, Russia, 1986 (Russian).

[20] H. Minc, "On permanents of circulants," Pacific Journal of Mathematics, vol. 42, pp. 477-484, 1972.

[21] H. Gupta, "Enumeration of incongruent cyclic $k$-gons," Indian Journal of Pure and Applied Mathematics, vol. 10, no. 8, pp. 964999, 1979.

[22] R. A. Brualdi and H. J. Ryser, Combinatorial Matrix Theory, vol. 39, Cambridge University Press, Cambridge, UK, 1991.

[23] V. S. Shevelev, "Some problems in the theory of permanents of cyclic matrices, problems 10-12," in Permanents: Theory and Applications, pp. 109-126, Polytechnic Institute, Krasnoyarsk, Russia, Collections of papers and problems, edited by G. P. Egorychev, 1990 (Russian). 


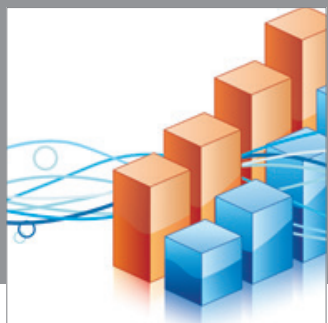

Advances in

Operations Research

mansans

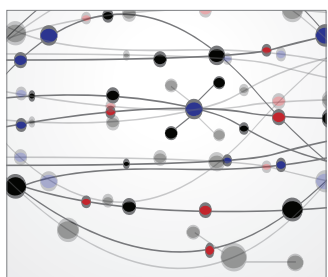

The Scientific World Journal
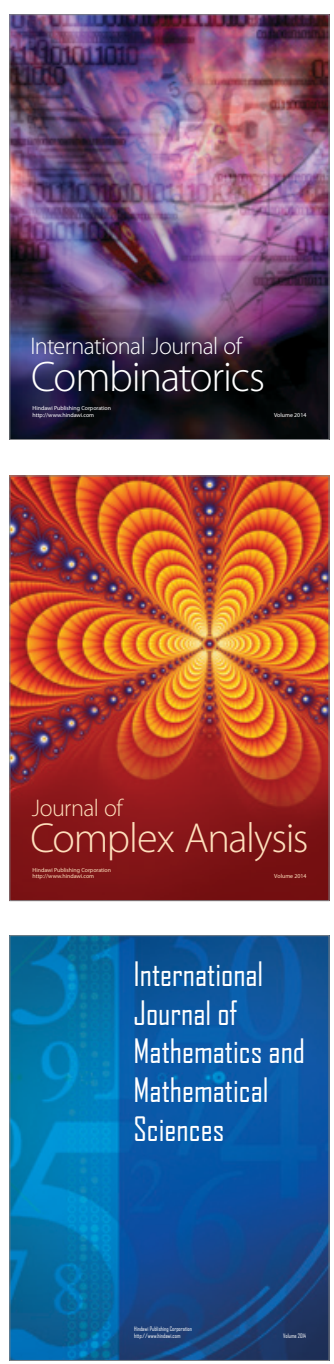
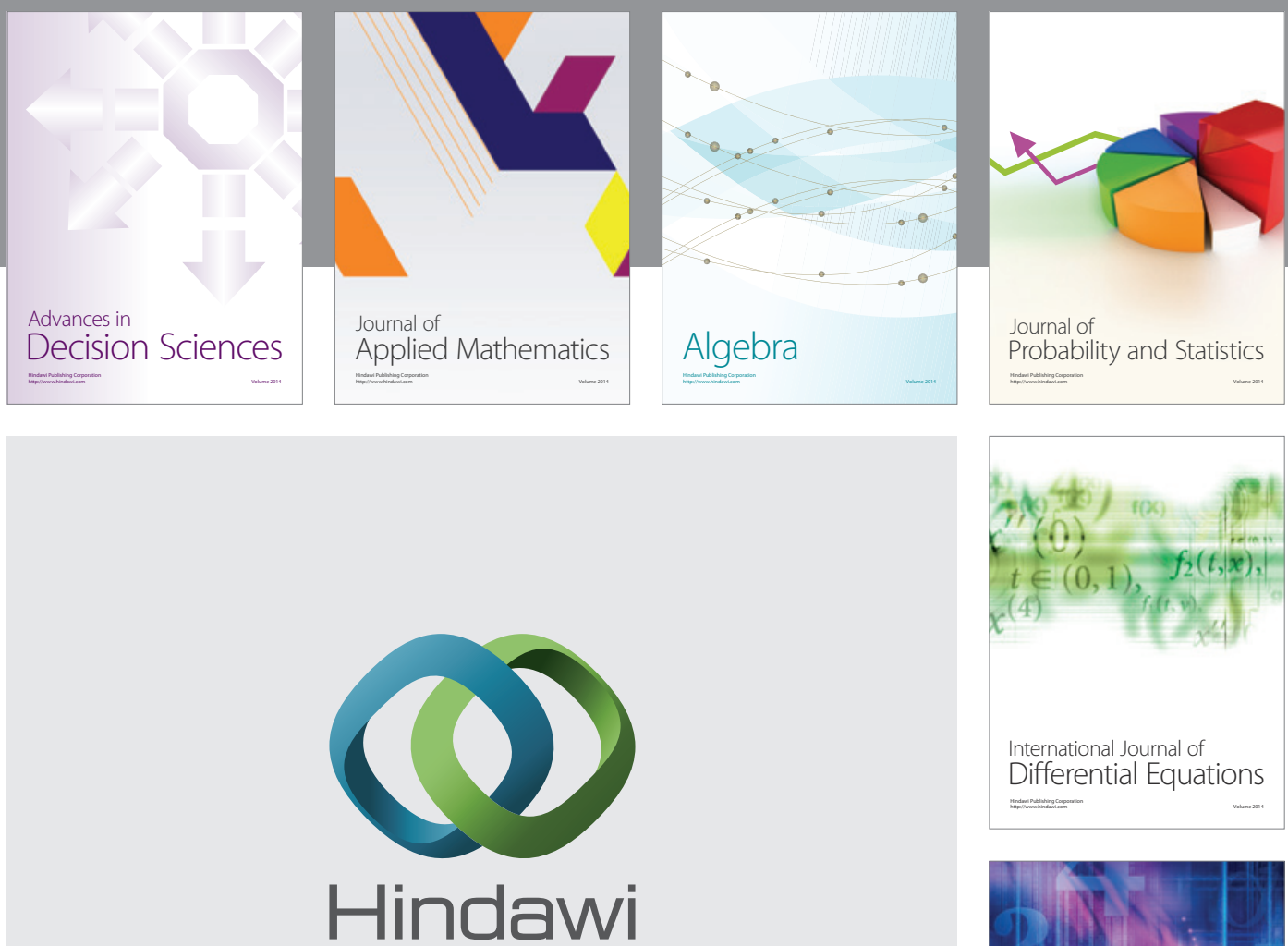

Submit your manuscripts at http://www.hindawi.com
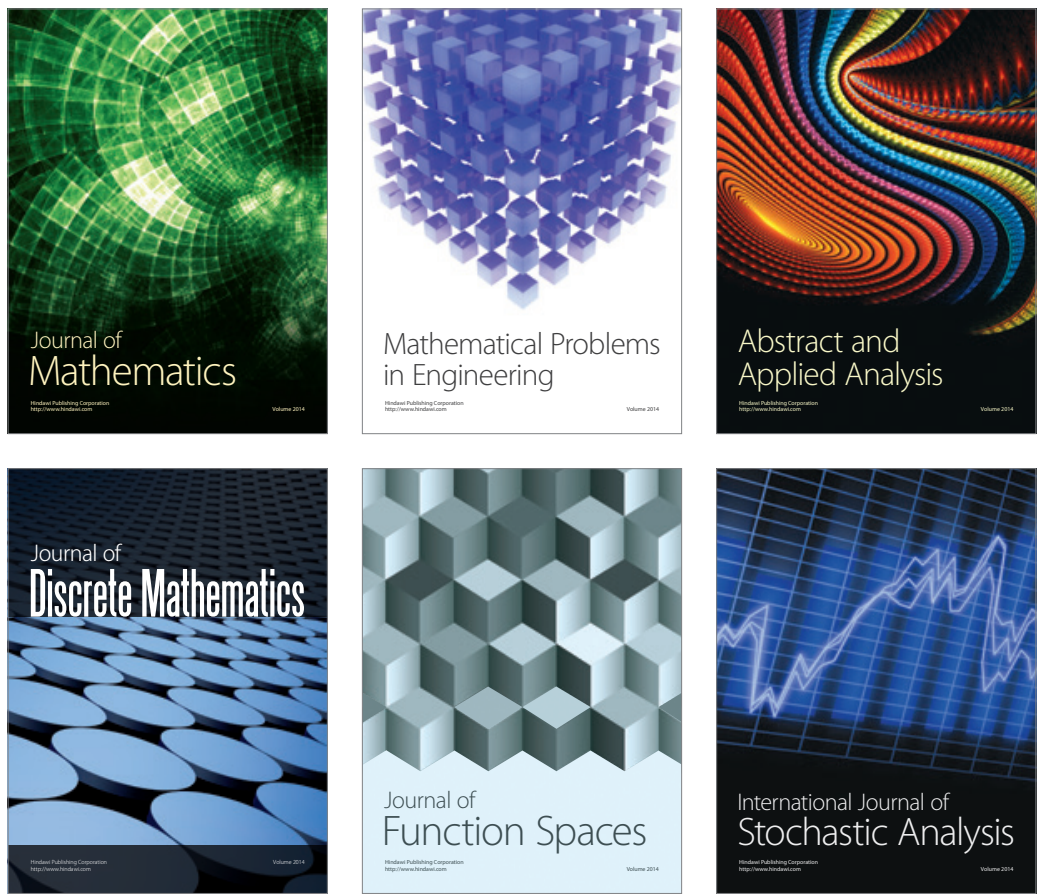

Journal of

Function Spaces

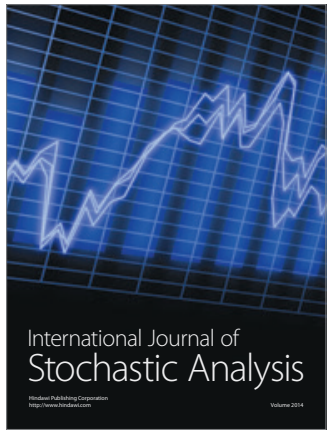

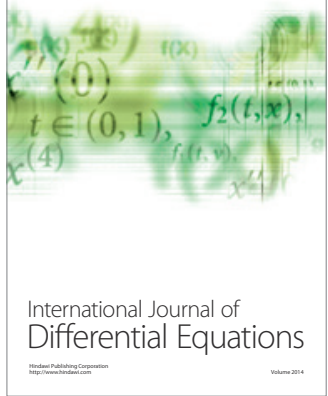
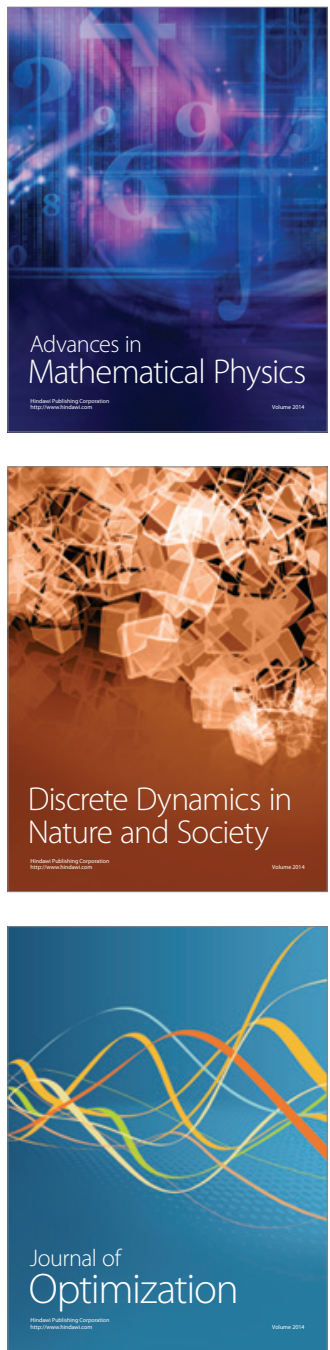